\title{
Bifurcation of local critical periods in the generalized Loud's system
}

\author{
J. Villadelprat \\ Departament de Matemàtica Aplicada i Anàlisi, \\ Universitat de Barcelona, \\ Gran Via 585, 08007 Barcelona, Spain
}

\begin{abstract}
We study the bifurcation of local critical periods in the differential system

$$
\left\{\begin{array}{l}
\dot{x}=-y+B x^{n-1} y, \\
\dot{y}=x+D x^{n}+F x^{n-2} y^{2},
\end{array}\right.
$$

where $B, D, F \in \mathbb{R}$ and $n \geqslant 3$ is a fixed natural number. Here by "local" we mean in a neighbourhood of the center at the origin. For $n$ even we show that at most two local critical periods bifurcate from a weak center of finite order or from the linear isochrone, and at most one local critical period from a nonlinear isochrone. For $n$ odd we prove that at most one local critical period bifurcates from the weak centers of finite or infinite order. In addition, we show that the upper bound is sharp in all the cases. For $n=2$ this was proved by Chicone and Jacobs in [Bifurcation of critical periods for plane vector fields, Trans. Amer. Math. Soc. 312 (1989) 433-486] and our proof strongly relies on their general results about the issue.
\end{abstract}

\section{Introduction and statement of the result}

In this paper we study the period function of centers of planar polynomial differential systems. A singular point of a planar differential system is a center if it has a punctured neighbourhood that consists entirely of periodic orbits surrounding it. The largest punctured neighbourhood with this property is called the period annulus of the center and, in what follows, it will be denoted by $\mathscr{P}$. Compactifying $\mathbb{R}^{2}$ to the Poincaré disc, the boundary of $\mathscr{P}$ has two connected components: the center itself and a polycycle. We call them respectively the inner and outer boundary of the period annulus. The period function of the center assigns to each periodic orbit $\gamma$ in $\mathscr{P}$ its period. If all the periodic orbits in $\mathscr{P}$ have the same period, then the center is called isochronous. Since the period function is defined on the set of periodic orbits in $\mathscr{P}$, usually the first step is to parameterize this set, let us say $\left\{\gamma_{s}\right\}_{s \in(0,1)}$, and then one can study the qualitative properties of the period function by means of the map $s \longmapsto$ period of $\gamma_{s}$, which is analytic on $(0,1)$. The critical periods are the critical points of this function and their number, character (maximum or minimum) and distribution do not depend on the particular parameterization of the set of periodic orbits used. We are interested in the bifurcation of critical periods. Roughly speaking, the disappearance or emergence of critical periods as we perturb the system. There are three different situations to study (see [13] for details):

(a) Bifurcation of the period function from the inner boundary of $\mathscr{P}$ (i.e., the center itself).

(b) Bifurcation of the period function from $\mathscr{P}$.

2010 AMS Subject Classification: 34C07; 34C23; 37C25.

Key words and phrases: center, period function, critical period, bifurcation.

The author is partially supported by the CONACIT through 2009-SGR-410 and by the DGES through MTM-2008-03437. 
(c) Bifurcation of the period function from the outer boundary of $\mathscr{P}$ (i.e., the polycycle).

In this paper we are only concerned with bifurcation of the period function from the inner boundary of $\mathscr{P}$, i.e. case $(a)$, and so when we talk about local critical period, see Definition 2.1, we mean in a neighbourhood of the center. This problem, as many others dealing with the period function and critical periods, is the counterpart of a problem about the Poincaré return map and limit cycles. More concretely, the problem of studying the cyclicity of a singular point by means of the Lyapunov coefficients, which is itself related with the Hilbert's 16th problem (see $[1,4,11]$ and references therein). In both cases the first stage is to study an algebraic variety for solving, respectively, the center-focus and isochronicity problems. Next it requires the knowledge of the ideal generated by the Lyapunov and period coefficients, respectively.

This paper strongly relies on the results of Chicone and Jacobs in [3], that constitutes the seminal work about the issue. Among other general results, in that excellent paper the authors give a complete description of the bifurcation of local critical periods in the quadratic systems. There are four families of quadratic centers: Hamiltonian, reversible, codimension four and generalized Lotka-Volterra systems. They show that the bifurcation of local critical periods can only occur inside the family of reversible systems, which can be brought by means of a linear change of variables to the form

$$
\left\{\begin{array}{l}
\dot{x}=-y+B x y \\
\dot{y}=x+D x^{2}+F y^{2}
\end{array}\right.
$$

In the literature this normal form is commonly known as Loud's system. This name stands for W. S. Loud, who proves in [12] that the center at the origin of system (1) is isochronous if, and only if, either $B=D=$ $F=0$, or $B \neq 0$ and $\left(\frac{D}{B}, \frac{F}{B}\right) \in\left\{i_{1}, i_{2}, i_{3}, i_{4}\right\}$, where

$$
i_{1}=(0,1), i_{2}=\left(-\frac{1}{2}, 2\right), i_{3}=\left(-\frac{1}{2}, \frac{1}{2}\right) \text { and } i_{4}=\left(0, \frac{1}{4}\right) .
$$

The isochrones are weak centers of infinite order, see Definition 2.2, and the study of their perturbations is the hardest part of the problem. Chicone and Jacobs [3, Theorem 3.1] prove for quadratic systems that at most two local critical periods bifurcate from a weak center of finite order or from the linear isochrone, and at most one local critical period from the nonlinear isochrones. In all the cases they show that the upper bound is sharp.

In the present paper we study the bifurcation of local critical periods in the planar differential system

$$
\left\{\begin{array}{l}
\dot{x}=-y+B x^{n-1} y, \\
\dot{y}=x+D x^{n}+F x^{n-2} y^{2},
\end{array}\right.
$$

where $B, D, F \in \mathbb{R}$ and $n \geqslant 3$. For obvious reasons we call it generalized Loud's system. Setting

$$
i_{1}:=(0,1), i_{2}:=\left(-\frac{n-1}{n}, n\right) \text { and } i_{3}:=\left(-\frac{1}{2}, \frac{1}{2}\right),
$$

the results that we obtain are summarized in the following two theorems:

Theorem A. Consider the center at the origin of system (2) with an even $n \geqslant 4$. Then the following hold:

(a) The center is isochronous if, and only if, either the system is linear or $B \neq 0$ and $\left(\frac{D}{B}, \frac{F}{B}\right) \in\left\{i_{1}, i_{2}, i_{3}\right\}$.

(b) A weak center which is not an isochrone has at most order two. Moreover, there are perturbations of the weak centers of order one with one local critical period, and there are perturbations of the weak centers of order two with exactly one or exactly two local critical periods.

(c) At most one local critical period bifurcates from a nonlinear isochrone, and at most two local critical periods bifurcate from the linear isochrone. Moreover, there are perturbations with exactly one local critical period from each nonlinear isochrone, and perturbations with exactly one or exactly two local critical periods from the linear isochrone. 
Theorem B. Consider the center at the origin of system (2) with an odd $n \geqslant 3$. Then the following hold:

(a) The center is isochronous if, and only if, either the system is linear or $B \neq 0$ and $\left(\frac{D}{B}, \frac{F}{B}\right) \in\left\{i_{1}, i_{2}\right\}$.

(b) A weak center which is not an isochrone has at most order one. Moreover, there are perturbations of the weak centers of order one with one local critical period.

(c) At most one local critical period bifurcates from an isochrone. Moreover, there are perturbations with exactly one local critical period from each isochrone.

The result for $n=2$ is exactly the same as Theorem A except for the fact that there exists a fourth isochrone placed at $i_{4}=\left(0, \frac{1}{4}\right)$. Apart from this, the case $n=2$ undergoes an additional degeneracy that makes more difficult the study carried out by Chicone and Jacobs in [3], see Remark 3.2. Let us also stress that their result holds for perturbations inside the whole family of quadratic centers, while our result is valid for perturbations inside the generalized Loud's system. This is natural because system (2) has arbitrary degree and the center-focus problem is "only" solved for polynomial systems of degree 2 or with homogenous nonlinearities of degree 3. Let us mention at this point that the results in Theorem $\mathrm{B}$ for $n=3$ are covered by the paper of Rousseau and Toni [15]. Indeed, in that paper the authors study the bifurcation of local critical periods from the centers of polynomial systems with homogeneous nonlinearities of third degree.

To tackle the problem of the bifurcation of local critical periods in a given family of polynomial centers we must first solve the isochronicity problem, i.e., to give the necessary and sufficient conditions for the center to be isochronous in terms of the coefficients of the system. For $n=2$ this follows from the classification of Loud [12]. As we will see, in our case it will be enough to have the sufficient conditions for the isochronicity of system (2) that are given in [2].

For other results concerning the bifurcation of local critical periods from the center the reader is referred to $[6,14,16]$ and references there in. Let us also mention that one may also consider bifurcation of critical periods from the period annulus (see $[8,10]$ ) or the polycycle in its outer boundery (see $[13,17]$ ).

The paper is organised in the following way. In Section 2 we give the definitions and preliminary results. In particular we compute some period coefficients of the center at the origin of system (2). This is done in Proposition 2.6, but for reasons of space we display them in an Appendix at the end of the paper. Section 3 is devoted to the proof of Theorem A. To be more precise, $(a)$ and $(b)$ follow from Theorem 3.1 and $(c)$ follows from Theorem 3.4. Finally Theorem B is proved in Section 4. More precisely, $(a)$ and $(b)$ follow from Theorem 4.1 and $(c)$ follows from Theorem 4.2. We conclude the paper with some observations for further research.

\section{Definitions and preliminary results}

As we have already mentioned, to study the period function of a center it is first necessary to parametrize the set of periodic orbits inside $\mathscr{P}$. To this end sometimes it is used a transversal section, but this causes the rather artificial property that only the even period coefficients are significant for the study of the bifurcation problem. In this paper, since we can compute a local first integral for system (2), see Lemma 2.4, we prefer to use its energy level to parametrize the periodic orbits instead of a transversal section. More generally, let $X_{\lambda}=P(x, y) \partial_{x}+Q(x, y) \partial_{y}$ with $\lambda \in \Lambda \subset \mathbb{R}^{k}$ be family of planar polynomial vector fields. (Here we suppose that the coefficients of $P$ and $Q$ are polynomials in $\lambda$.) Let us assume that the differential system associated to $X_{\lambda}$ has a non-degenerated center at the origin for all $\lambda \in \Lambda$. In this case we can suppose without loss of generality that the linearization of $X_{\lambda}$ at $(0,0)$ is $(x, y) \longmapsto(-y, x)$. Let $H_{\lambda}$ be an analytic local first integral of the center with $H_{\lambda}(x, y)=\frac{x^{2}+y^{2}}{2}+\mathrm{o}\left(x^{2}+y^{2}\right)$. Then the periodic orbits near the center are ovals inside $\left\{H_{\lambda}=h\right\}$ for positive $h \approx 0$. Let $P(h ; \lambda)$ be the period of the periodic orbit of $X_{\lambda}$ inside $\left\{H_{\lambda}=h\right\}$. It is well known that the function $h \longmapsto P(h ; \lambda)$ can be extended analytically to $h=0$ setting $P(0 ; \lambda)=2 \pi$ 
and that the coefficients of its Taylor series at $h=0$, the so-called period coefficients, are polynomial in $\lambda$ (see for instance $[3,5]$ and references therein).

For the differential system (2) we have $\lambda=(B, D, F)$ and $\Lambda=\mathbb{R}^{3}$. Furthermore, see Proposition 2.6 below, it turns out that $P(h ; \lambda)$ is analytic on $h^{\alpha}$ for some $\alpha \in \mathbb{N}$. (To be more precise, $\alpha=n-1$ for $n$ even and $\alpha=\frac{n-1}{2}$ for $n$ odd.) We have thus that its MacLaurin series is given by

$$
P(h ; \lambda)=2 \pi+\sum_{k \geqslant 1} p_{k}(\lambda) h^{\alpha k}
$$

where $p_{k} \in \mathbb{R}[\lambda]$ are the period coefficients of the center.

Definition 2.1. We say that $\ell$ local critical periods bifurcate from the center corresponding to the parameter value $\lambda_{*}$ if for every $\varepsilon>0$ and every neighbourhood $U$ of $\lambda_{*}$ there exists $\lambda_{0} \in U$ such that $P^{\prime}\left(h ; \lambda_{0}\right)=0$ has $\ell$ solutions on $(0, \varepsilon)$.

Definition 2.2. If $p_{1}\left(\lambda_{*}\right)=p_{2}\left(\lambda_{*}\right)=\ldots=p_{k}\left(\lambda_{*}\right)=0$ and $p_{k+1}\left(\lambda_{*}\right) \neq 0$ with $k \geqslant 1$, then the center at the origin corresponding to the parameter value $\lambda_{*}$ is called a weak center of order $k$. The center is isochronous if all its periodic orbits have the same period, i.e., $p_{k}\left(\lambda_{*}\right)=0$ for all $k \in \mathbb{N}$. In this case the center is also called a weak center of infinite order.

Let us point out that the number of local critical periods bifurcating from the center does not depend on the parametrization of the set of periodic orbits in $\mathscr{P}$ that we have chosen. Note in addition that local critical periods can only bifurcate from weak centers. An easy argument shows that at most $k$ local critical periods can bifurcate from a weak center of order $k$. The case of a weak center of infinite order, i.e., an isochrone, is much more delicate because usually it is necessary to analyze the ideal generated by all the period coefficients. To this end we introduce the following notations:

Definition 2.3. The algebraic variety defined by an ideal $\mathfrak{m}$ over the $\operatorname{ring} \mathbb{R}\left[x_{1}, x_{2}, \ldots, x_{n}\right]$ is

$$
V(\mathfrak{m})=\left\{z \in \mathbb{C}^{n}: f(z)=0 \text { for all } f \in \mathfrak{m}\right\} .
$$

Following [20] we denote by ${ }^{a} \mathfrak{m}$ the dehomogenization of an ideal $\mathfrak{m}$ in the $\operatorname{ring} \mathbb{R}[B, D, F]$ using $B$. Moreover, if $f \in \mathfrak{m}$ then we define ${ }^{a} f(D, F):=f(1, D, F)$. Finally, ${ }^{h} \mathfrak{m}$ denotes the homogenization of an ideal $\mathfrak{m}$ in $\mathbb{R}[D, F]$ using a third indeterminant $B$.

The rest of this section is devoted to prove some preliminary results that were in fact already used in the previous definitions. We begin by giving a local first integral of system (2), which shows in particular that the singular point at the origin is a non-degenerated center for all $(B, D, F) \in \mathbb{R}^{3}$.

Lemma 2.4. (a) The function

$$
\kappa(x):= \begin{cases}\left(1-B x^{n-1}\right)^{-1-\frac{2 F}{B(n-1)}} & \text { if } B \neq 0, \\ \exp \left(\frac{2 F}{n-1} x^{n-1}\right) & \text { if } B=0,\end{cases}
$$

is an integrating factor for system (2). The first integral is given by $H(x, y)=A(x)+C(x) y^{2}$ with

$$
A(x):=\int_{0}^{x}\left(s+D s^{n}\right) \kappa(s) d s \text { and } C(x):=\frac{1}{2}\left(1-B x^{n-1}\right) \kappa(x) .
$$

(b) The coordinate transformation $\{u=f(x), v=\sqrt{2 C(x)} y\}$, where

$$
f(x):=\int_{0}^{x} \frac{\kappa(s)}{\sqrt{2 C(s)}} d s,
$$


brings system (2) to the potential system

$$
\left\{\begin{array}{l}
\dot{u}=-v \\
\dot{v}=\left(A \circ f^{-1}\right)^{\prime}(u) .
\end{array}\right.
$$

Proof. The first part is a straightforward computation and the second one follows from [7, Lemma 5].

Lemma 2.5. Suppose that $f(x)=x \hat{f}\left(x^{k}\right)$, where $k \in \mathbb{N}$ and $\hat{f}$ is analytic at $x=0$ with $\hat{f}(0)=1$. Then $f$ is a diffeomorphism near $x=0$ and $f^{-1}(x)=x \hat{g}\left(x^{k}\right)$, where $\hat{g}$ is analytic at $x=0$ with $\hat{g}(0)=1$. Finally, if the MacLaurin series for $\hat{f}$ is $\hat{f}(x)=1+\sum_{n \geqslant 1} \alpha_{n} x^{n}$, then the first six coefficients in $\hat{g}(x)=1+\sum_{n \geqslant 1} \beta_{n} x^{n}$ are the ones given in the Appendix.

Proof. That $f$ is a diffeomorphism is obvious. The existence of $\hat{g}$ such that $f^{-1}(x)=x \hat{g}\left(x^{k}\right)$ follows from noting that $\left(f \circ f^{-1}\right)(x)=x$ is equivalent to $\hat{g}(u) \hat{f}\left(u \hat{g}(u)^{k}\right)=1$, where $u=x^{k}$. Then, by applying the implicit function theorem to $R(u, v):=v \hat{f}\left(u v^{k}\right)-1$, we can assert that there exists an analytic function $\hat{g}$ such that $R(u, \hat{g}(u))=0$ for all $u \approx 0$. Standard computations lead to the coefficients in the Appendix.

From now on $\lambda:=(B, D, F) \in \mathbb{R}^{3}$ and $P(h ; \lambda)$ is the period of the periodic orbit of $(2)$ inside $\{H=h\}$, where $H$ is the first integral given in Lemma 2.4. The next result provides some properties of the function $h \longmapsto P(h ; \lambda)$. In particular it shows that the period coefficient $p_{k}$, modulo $\left(p_{1}, p_{2}, \ldots, p_{k-1}\right)$ in $\mathbb{R}[\lambda]$, is a homogeneous polynomial of degree $k$. (Here, and from now on where there is no risk of ambiguity, we omit the dependence on $\lambda$.)

Proposition 2.6. Let $g(u):=\operatorname{sgn}(u) \sqrt{2 A \circ f^{-1}}(u)$, with $A$ and $f$ given by Lemma 2.4. Then:

(a) $g$ is a diffeomorphism at $x=0$ and $g^{-1}(u)=u \hat{g}\left(u^{n-1}\right)$ with $\hat{g}$ analytic at $u=0$ and $\hat{g}(0)=1$.

(b) If the MacLaurin series for $\left(g^{-1}\right)^{\prime}$ is given by $\left(g^{-1}\right)^{\prime}(u)=1+\sum_{k \geqslant 1} d_{k}(\lambda) u^{k(n-1)}$, then

$$
P(h ; \lambda)=2 \pi+\sum_{k \geqslant 1} p_{k}(\lambda) h^{\frac{k(n-1)}{2}},
$$

where $p_{k}(\lambda)=\left(1+(-1)^{k(n-1)}\right) \beta_{k} d_{k}(\lambda)$ with $\beta_{k}$ being a positive number.

(c) For all $k, p_{k}=\hat{p}_{k}+q_{k}$, where $\hat{p}_{k} \in \mathbb{R}[\lambda]$ is homogeneous of degree $k$ and $q_{k} \in\left(p_{1}, p_{2}, \ldots, p_{k-1}\right)$.

(d) The expression of $d_{1}, d_{2}, d_{3}, d_{4}$ and $d_{6}$ are given in the Appendix.

Proof. For the sake of shortness, let us say that $s \in \mathcal{I}$ when $s(x)$ is an analytic function at $x=0$ with $s(0)=1$. Thus $A^{\prime}(x)=x s_{1}\left(x^{n-1}\right)$ and $f^{\prime}(x)=s_{2}\left(x^{n-1}\right)$ with $s_{i} \in \mathcal{I}$. Therefore $A(x)=\frac{1}{2} x^{2} s_{3}\left(x^{n-1}\right)$, $f(x)=x s_{4}\left(x^{n-1}\right)$ and, by applying Lemma 2.5, $f^{-1}(x)=x s_{5}\left(x^{n-1}\right)$, where $s_{i} \in \mathcal{I}$. Thus, if we define $V:=A \circ f^{-1}$, then $V(u)=\frac{1}{2} u^{2} s_{6}\left(u^{n-1}\right)$ with $s_{6} \in \mathcal{I}$. Accordingly $g(u)=\operatorname{sgn}(u) \sqrt{2 V(u)}=u \sqrt{s_{6}\left(u^{n-1}\right)}$ and so, by Lemma 2.5 again, $g^{-1}(u)=u \hat{g}\left(u^{n-1}\right)$ with $\hat{g} \in \mathcal{I}$. This proves statement $(a)$. Thus the MacLaurin series of the derivative of $g^{-1}$ writes as

$$
\left(g^{-1}\right)^{\prime}(u)=1+\sum_{k \geqslant 1} d_{k} u^{k(n-1)} .
$$

The coordinate transformation in $(b)$ of Lemma 2.4 preserves the energy level of the periodic orbits, i.e., it maps the periodic orbit of (2) inside $A(x)+C(x) y^{2}=h$ to the periodic orbit of $(3)$ inside $\frac{1}{2} v^{2}+V(u)=h$. Taking this into account, by applying [3, Lemma 4.1] we can assert that

$$
P(h)=2 \int_{-\pi / 2}^{\pi / 2}\left(g^{-1}\right)^{\prime}(\sqrt{2 h} \sin \theta) d \theta,
$$


and consequently, from (4),

$$
P(h)=2 \pi+2 \sum_{k \geqslant 1} d_{k}\left(\int_{-\pi / 2}^{\pi / 2} \sin ^{k(n-1)} \theta d \theta\right)(2 h)^{\frac{k(n-1)}{2}} .
$$

Since each integral is equal to zero if, and only if, $k(n-1)$ is odd, and $\int_{-\pi / 2}^{\pi / 2} \sin ^{2 m} \theta d \theta=\frac{\pi(2 m) !}{4^{m}(m !)^{2}}$, some computations show that $P(h ; \lambda)=2 \pi+\sum_{k \geqslant 1} p_{k}(\lambda) h^{\frac{k(n-1)}{2}}$ where $p_{k}(\lambda)=\left(1+(-1)^{k(n-1)}\right) \beta_{k} d_{k}(\lambda)$ and

$$
\beta_{k}=\frac{\pi(k(n-1)) !}{2^{\frac{k(n-1)}{2}}\left(\left(\frac{k(n-1)}{2}\right) !\right)^{2}} .
$$

This shows $(b)$. In order to prove $(c)$, let $\widehat{P}(x)$ be the period of the periodic orbit of (2) passing through the point $(x, 0)$. Then $\widehat{P}(x)=2 \pi+\sum_{k \geqslant 2} \hat{p}_{k} x^{k}$ and, by applying [5, Proposition 12], we can assert that the coefficient $\hat{p}_{k(n-1)}$, modulo the ideal generated by the previous ones in $\mathbb{R}[\lambda]$, is a homogeneous polynomial of degree $k$. On account of this, $(c)$ follows from $(b)$ and the fact that $\widehat{P}(x)=P(V(x))$ with $V(x)=\frac{1}{2} x^{2}+\mathrm{o}\left(x^{2}\right)$.

Let us explain finally how we obtain the coefficients in $(d)$. To this end we first compute the MacLaurin series of $V$ by using that $V^{\prime \prime}=\left(\frac{A^{\prime \prime} f^{\prime}-A^{\prime} f^{\prime \prime}}{\left(f^{\prime}\right)^{3}}\right) \circ f^{-1}$, where some computations show that

$$
\left(\frac{A^{\prime \prime} f^{\prime}-A^{\prime} f^{\prime \prime}}{\left(f^{\prime}\right)^{3}}\right)(x)=1+(F-B+D n) x^{n-1}+D(F-B n) x^{2(n-1)} .
$$

For $B \neq 0$ one can also verify that

$$
f(x)=\int_{0}^{x} \frac{d s}{\left(1-B s^{n-1}\right)^{1+\frac{F}{B(n-1)}}}=x\left(1+\sum_{k \geqslant 1} \frac{(-B)^{k}}{k(n-1)+1}\left(\begin{array}{l}
\gamma \\
k
\end{array}\right) x^{k(n-1)}\right)
$$

with $\gamma=-1-\frac{F}{B(n-1)}$. Then Lemma 2.5 provides the first six coefficients in the MacLaurin series of the function $s_{5}$ verifying that $f^{-1}(u)=u s_{5}\left(u^{n-1}\right)$. Next, from (5),

$$
V^{\prime \prime}(u)=1+(F-B+D n) u^{n-1}\left(s_{5}\left(u^{n-1}\right)\right)^{n-1}+D(F-B n) u^{2(n-1)}\left(s_{5}\left(u^{n-1}\right)\right)^{2(n-1)},
$$

and we get the first six coefficients of the power series expansion of the function $s_{7}$ in $V^{\prime \prime}(u)=s_{7}\left(u^{n-1}\right)$. Then by integrating twice this series we obtain the first six coefficients of the Taylor's development of the function $s_{6}$ in $V(u)=\frac{1}{2} u^{2} s_{6}\left(u^{n-1}\right)$. This will allow us to compute the first six coefficients of the power series expansion of the function $\hat{g}$ in $g^{-1}(u)=u \hat{g}\left(u^{n-1}\right)$. Indeed, note that by definition it holds $V\left(g^{-1}(u)\right)=u^{2}$, and so we can obtain the coefficients in the expansion of $\hat{g}$ by imposing that

$$
\frac{1}{2} u^{2}\left(\hat{g}\left(u^{n-1}\right)\right)^{2} s_{6}\left(u^{n-1}\left(\hat{g}\left(u^{n-1}\right)\right)^{n-1}\right)=u^{2} \text { for all } u .
$$

In this way we find the expression of the first coefficients in $\left(g^{-1}\right)^{\prime}(u)=1+\sum_{k \geqslant 1} d_{k} u^{k(n-1)}$ that appear in the Appendix. (In principle the formulas that we obtain are valid for $B \neq 0$. Their validity for $B=0$ is clear because $d_{k} \in \mathbb{R}[\lambda]$.) This completes the proof of the result.

\section{Proof of Theorem A}

In this case, since $n$ is even, from Proposition 2.6 it follows that the MacLaurin series of the period function is given by

$$
P(h)=2 \pi+\sum_{k \geqslant 1} p_{2 k} h^{k(n-1)} .
$$


Thus the center is isochronous if, and only if, $p_{2 k}=0$ for all $k \in \mathbb{N}$. The following result shows that the vanishing of the period coefficients $p_{2}, p_{4}$ and $p_{6}$ already determines the isochrones. In its statement, and from now on, we use the notation introduced in Definition 2.3.

Theorem 3.1. Consider the differential system (2) with an even $n \geqslant 4$. Then the following hold:

(a) The center is isochronous if, and only if, either the system is linear or $B \neq 0$ and $\left(\frac{D}{B}, \frac{F}{B}\right) \in\left\{i_{1}, i_{2}, i_{3}\right\}$.

(b) If $B=0$, then there are not weak centers of finite order. If $B \neq 0$, then a weak center which is not an isochrone has at most order two. Moreover there are perturbations of the weak centers of order one with one critical period, and there are perturbations of the weak centers of order two with exactly one or exactly two critical periods.

(c) If $\mathfrak{m}_{1}=\left(p_{2}, p_{4}\right)$ and $\mathfrak{m}_{2}=\left(p_{2}, p_{4}, p_{6}\right)$, then ${ }^{a} \mathfrak{m}_{1}$ and ${ }^{a} \mathfrak{m}_{2}$ are radical in $\mathbb{R}[D, F]$.

Proof. Note first (see the Appendix) that $p_{2}, p_{4}$ and $p_{6}$ are homogeneous polynomials in $B, D$ and $F$ of degrees 2,4 and 6 , respectively. If $B=0$ then we have that (up to a positive factor)

$$
p_{2}=(5 n+2) F^{2}+\left(8 n^{2}-2\right) F D+\left(4 n^{3}-n\right) D^{2}
$$

and it is easy to show that $D=F=0$ is the unique real root of this polynomial. This proves the assertions in $(a)$ and $(b)$ concerning the case $B=0$. Let us turn next to the case $B \neq 0$. Note first that we can take $B=1$ due to the homogeneity properties of the period coefficients. By Bezout's theorem ${ }^{a} p_{2}$ and ${ }^{a} p_{4}$ can have at most eight common zeros counted with multiplicities over pairs of complex numbers. To determine them we take advantage of the elimination property of the Gröbner bases computed relative to the lexicographic order. More concretely, we compute the Gröbner basis of ${ }^{a} \mathfrak{m}_{1}=\left({ }^{a} p_{2},{ }^{a} p_{4}\right)$ with respect to the monomial order $B>F>D$. We thus obtain that ${ }^{a} \mathfrak{m}_{1}=\left(q_{1}, q_{2}\right)$, with

$$
q_{1}(D)=D(2 D+1)(n D+n-1) r_{1}(D) \text { and } q_{2}(D, F)=\eta F+r_{2}(D),
$$

where $\eta \in \mathbb{R}[n]$ and $r_{i} \in \mathbb{R}[D, n]$. More concretely, $r_{1}$ is the fifth degree polynomial

$$
\begin{aligned}
r_{1}(D)= & 8(2 n-1)\left(13273 n^{5}-8958 n^{4}-2531 n^{3}+2238 n^{2}-14 n-84\right) D^{5} \\
& +4(2 n-1)\left(86105 n^{5}-66192 n^{4}-8557 n^{3}+11640 n^{2}+68 n-528\right) D^{4} \\
& +2(2 n-1)\left(178765 n^{5}-160482 n^{4}-2111 n^{3}+23952 n^{2}-632 n-1224\right) D^{3} \\
& +\left(-30976 n^{2}+652 n-411469 n^{5}+247654 n^{6}+193684 n^{4}+1344+25483 n^{3}\right) D^{2} \\
& +\left(-3099 n^{3}+1497 n+42627 n^{4}+7302 n^{6}-9843 n^{2}+354-27942 n^{5}\right) D \\
& -18(n-2)(3 n-1)\left(2 n^{2}-11 n-1\right)(n+1)^{2} .
\end{aligned}
$$

(It is important to note that, although $n$ is fixed, we must check that all the computations are valid for any even $n \geqslant 4$. For instance, to conclude that ${ }^{a} \mathfrak{m}_{1}=\left(q_{1}, q_{2}\right)$ we use that the polynomials ${ }^{a} p_{1}$ and ${ }^{a} p_{2}$ write as ${ }^{a} p_{i}=\left(a_{i} q_{1}+b_{i}, q_{2}\right) / c$ for $i=1,2$, where $c \in \mathbb{R}[n]$ having $n=2$ as its unique natural root and $\left.a_{i}, b_{i} \in \mathbb{R}[D, F, n].\right)$

Since one can verify that $n=2$ is the only natural root of $\eta=0$, the common zeros of ${ }^{a} p_{2}$ and ${ }^{a} p_{4}$ are pairs $(D, F)$ such that $q_{1}(D)=0$ and $F=-r_{2}(D) / \eta$. Three of these roots are given by

$$
i_{1}=(0,1), \quad i_{2}=\left(-\frac{n-1}{n}, n\right) \text { and } i_{3}=\left(-\frac{1}{2}, \frac{1}{2}\right) \text {. }
$$

By Theorems 2.1 and 2.2 in $[2]$, if $\left(\frac{D}{B}, \frac{F}{B}\right) \in\left\{i_{1}, i_{2}, i_{3}\right\}$, then the center at the origin of system (2) is isochronous. Accordingly, for each $k=1,2,3$, we have that ${ }^{a} p_{2 m}\left(i_{k}\right)=0$ for all $m \in \mathbb{N}$. Next we shall show that these are the only isochrones. Let $\widehat{D}_{i}$ for $i=1,2, \ldots, 5$ be the complex roots of $r_{1}(D)=0$, and define $\ell_{i}=\left(\widehat{D}_{i}, \widehat{F}_{i}\right)$ with $\widehat{F}_{i}=-r_{2}\left(\widehat{D}_{i}\right) / \eta$. In particular,

$$
V\left({ }^{a} \mathfrak{m}_{1}\right)=\left\{i_{1}, i_{2}, i_{3}, \ell_{1}, \ell_{2}, \ell_{3}, \ell_{4}, \ell_{5}\right\}
$$




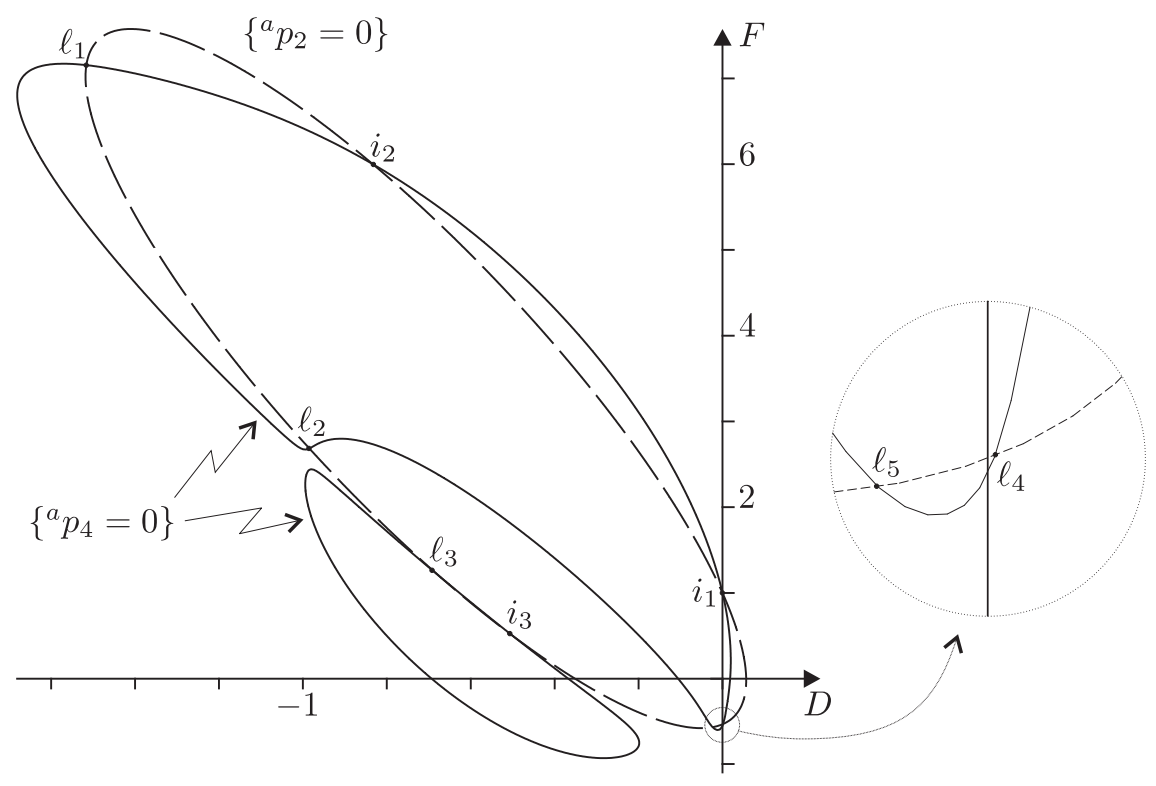

Figure 1: Location of the weak centers of system (2) for $n=6$.

By computing the discriminant of $r_{1}$ with respect to $D$ we can assert that the number of its real roots for $n>3$ can only change at $n \approx 4.72$. (Here we took into account that its leading coefficient does not vanish for $n>3$.) Next, by applying Sturm's algorithm we conclude that $r_{1}$ has three real roots for $n=4$ and five real roots for $n \geqslant 6$. Moreover all of them are simple and such that $\widehat{D}_{i} \notin\left\{0,-\frac{n-1}{n},-\frac{1}{2}\right\}$ for all $i=1,2, \ldots, 5$. We claim that ${ }^{a} p_{6}\left(\ell_{i}\right) \neq 0$ for all $i=1,2, \ldots, 5$. This will prove that each $\ell_{i} \in \mathbb{R}^{2}$ is a weak center of order two and that $i_{1}, i_{2}$ and $i_{3}$ are the only isochrones. To show the claim we compute the resultant between $r_{1}(D)$ and ${ }^{a} p_{6}\left(D,-r_{2}(D) / \eta\right)$. The resultant is a polynomial in $n$ and one can verify that $n=2$ is its only natural root. So the claim is true and statement $(a)$ follows. Therefore,

$$
V\left({ }^{a} \mathfrak{m}_{2}\right)=\left\{i_{1}, i_{2}, i_{3}\right\}
$$

Next we claim that the Jacobian matrix of the mapping $\mathscr{C}(D, F):=\left({ }^{a} p_{2}(D, F),{ }^{a} p_{4}(D, F)\right)$ has rank 2 at each point in $V\left({ }^{a} \mathfrak{m}_{1}\right)$. Indeed, denoting the determinant of the Jacobian matrix by $K(D, F)$, the claim is true because one can check that the resultant between $q_{1}(D)$ and $K\left(D,-r_{2}(D) / \eta\right)$ is different from zero. Then by the Ideal Membership Theorem in [3, Theorem A.1] it follows that ${ }^{a} \mathfrak{m}_{1}$ is radical. In fact, since the claim also implies that the Jacobian matrix of $(D, F) \longmapsto\left({ }^{a} p_{2}(D, F),{ }^{a} p_{4}(D, F),{ }^{a} p_{6}(D, F)\right)$ has rank 2 at each point in $V\left({ }^{a} \mathfrak{m}_{2}\right)$, by applying the same result we conclude that ${ }^{a} \mathfrak{m}_{2}$ is radical too. This proves $(c)$.

Note finally that $\mathscr{C}$ is a local diffeomorphism at each $\ell_{i}$. This easily implies (for instance, by applying the Weierstrass Preparation Theorem) that there are perturbations of $\ell_{i}$ with exactly one or exactly two critical periods. This shows the last assertion in $(b)$ and concludes the proof of the result.

Remark 3.2. Figure 1 displays a numerical plot of the curves ${ }^{a} p_{2}=0$ and ${ }^{a} p_{4}=0$ for $n=6$ with the location of the weak centers. There are five of order two, $\ell_{1}, \ell_{2}, \ldots, \ell_{5}$, and three isochrones, $i_{1}, i_{2}, i_{3}$. As we explain in the proof of Theorem 3.1, this distribution holds for any even $n \geqslant 6$. For $n=4$ it turns out that $\ell_{4}$ and $\ell_{5}$ are not real. For $n=2$ it occurs that the weak center of order two at $\ell_{4}$ becomes isochrone, the fourth one placed at $i_{4}=\left(0, \frac{1}{4}\right)$. Moreover, $\nabla^{a} p_{2}$ and $\nabla^{a} p_{4}$ are not linearly independent at $i_{3}$ anymore because $\ell_{3}$ collides with it. This makes that the analysis of the ideals ${ }^{a} \mathfrak{m}_{1}$ and ${ }^{a} \mathfrak{m}_{2}$ more complicated than the case $n \geqslant 4$. In particular they are not radical. 
By Theorem $3.1,{ }^{a} p_{2 k}$ vanishes at $V\left({ }^{a} \mathfrak{m}_{2}\right)=\left\{i_{1}, i_{2}, i_{3}\right\}$ for all $k$, and this plays an important role in the proof of the next result. This is the reason why the sufficient conditions for isochronicity given in [2] are crucial for our purposes. We remark that our proof follows almost verbatim the one of [3, Theorem 3.8].

Proposition 3.3. The period coefficients for the center at the origin of system (2) with an even $n \geqslant 4$ are in the ideal $\mathfrak{m}_{2}=\left(p_{2}, p_{4}, p_{6}\right)$ over the ring $\mathbb{R}[B, D, F]$.

Proof. By Theorem 3.1, ${ }^{a} \mathfrak{m}_{2}$ is radical and $V\left({ }^{a} \mathfrak{m}_{2}\right)=\left\{i_{1}, i_{2}, i_{3}\right\}$. Since the Jacobian matrix of the mapping $(D, F) \longmapsto\left({ }^{a} p_{2}(D, F),{ }^{a} p_{4}(D, F),{ }^{a} p_{6}(D, F)\right)$ has rank 2 at the points $i_{1}, i_{2}$ and $i_{3}$, by the Exponent One Lemma in [3, Lemma A.1], the exponents in the primary decomposition of ${ }^{a} \mathfrak{m}_{2}$ at $i_{1}, i_{2}$ and $i_{3}$ are all one. By Noether's Fundamental Theorem (see [18, pages 61-65]), this implies that ${ }^{a} \mathfrak{m}_{2}=\mathfrak{q}_{1} \cap \mathfrak{q}_{2} \cap \mathfrak{q}_{3}$, where

$$
\begin{aligned}
& \mathfrak{q}_{1}:=\left({ }^{a} \mathfrak{m}_{2}, D, F-1\right)=(D, F-1), \\
& \mathfrak{q}_{2}:=\left({ }^{a} \mathfrak{m}_{2}, n D+n-1, F-n\right)=(n D+n-1, F-n), \\
& \mathfrak{q}_{3}:=\left({ }^{a} \mathfrak{m}_{2}, 2 D+1,2 F-1\right)=(2 D+1,2 F-1) .
\end{aligned}
$$

Then (see [20, pages 180-182]) it follows that $\mathfrak{m}_{2} \subset{ }^{h}\left({ }^{a} \mathfrak{m}_{2}\right)={ }^{h} \mathfrak{q}_{1} \cap{ }^{h} \mathfrak{q}_{2} \cap{ }^{h} \mathfrak{q}_{3}$ and

$$
{ }^{h} \mathfrak{q}_{1}=(D, F-B), \quad{ }^{h} \mathfrak{q}_{2}=(n D+(n-1) B, F-n B) \text { and }{ }^{h} \mathfrak{q}_{3}=(2 D+B, 2 F-B)
$$

Since each ${ }^{h} \mathfrak{q}_{i}$ is generated by two elements without non-constant common divisor for $B=0$, it follows from [18, pages 64-65] that a homogeneous polynomial $f(B, D, F)$ belongs to the ideal ${ }^{h}\left({ }^{a} \mathfrak{m}_{2}\right)$ if, and only if, $f(1, D, F) \in{ }^{a} \mathfrak{m}_{2}$. Since ${ }^{a} p_{2 k}$ vanishes at $V\left({ }^{a} \mathfrak{m}_{2}\right)=\left\{i_{1}, i_{2}, i_{3}\right\}$ for all $k \in \mathbb{N}$, and ${ }^{a} \mathfrak{m}_{2}$ is radical, this implies that $p_{2 k} \in{ }^{h}\left({ }^{a} \mathfrak{m}_{2}\right)$ for all $k \in \mathbb{N}$. Define at this point $\mathfrak{i}_{0}=\left(\mathfrak{m}_{2}, B^{6}\right)$. One can verify (for instance, by means of the Gröbner package of Maple) that $\mathfrak{m}_{2}={ }^{h} \mathfrak{q}_{1} \cap{ }^{h} \mathfrak{q}_{2} \cap{ }^{h} \mathfrak{q}_{3} \cap \mathfrak{i}_{0}$. Consequently,

$$
\mathfrak{m}_{2}={ }^{h}\left({ }^{a} \mathfrak{m}_{2}\right) \cap \mathfrak{i}_{0}
$$

We claim that $p_{2 k} \in \mathfrak{i}_{0}$ for all $k \geqslant 4$. The result will follow once we prove this because $p_{2 k} \in{ }^{h}\left({ }^{a} \mathfrak{m}_{2}\right)$ for all $k \in \mathbb{N}$. To show the claim we shall take advantage of $(c)$ in Proposition 2.6, which shows that $p_{2 k}$, modulo $\left(p_{2}, p_{4}, \ldots, p_{2(k-1)}\right)$ in $\mathbb{R}[B, D, F]$, is a homogeneous polynomial of degree $2 k$. Therefore to prove the claim it suffices to show that $B^{i} D^{j} F^{k} \in \mathfrak{i}_{0}$ for all $i, j, k \in \mathbb{N}$ such that $i+j+k=8$. There are 45 of these monomials and (by using once again the Gröbner package of Maple) one can readily check that they belong to $\mathfrak{i}_{0}$. (The coefficients in the normal form of each monomial in a Gröbner basis of $\mathfrak{i}_{0}$ are of the form $p / q$ where $p \in \mathbb{R}[B, D, F, n]$ and $q \in R[n]$. We have verified that no $q$ in the denominator vanishes at an even number greater than 2.) This concludes the proof of the result.

On account of [3, Theorem 3.8], the previous result is true for $n=2$ as well. In that case, since there exists a fourth isochrone, the primary decomposition of ${ }^{a} \mathfrak{m}_{2}$ has an additional term, $\mathfrak{q}_{4}$. On the other hand, for $n=2$ it suffices to take $\mathfrak{i}_{0}=\left(\mathfrak{m}_{2}, B^{4}\right)$ and to show that every monomial of degree seven is in $\mathfrak{i}_{0}$. Apart from working with an arbitrary $n$, these are the unique differences between the proofs of Proposition 3.3 and [3, Theorem 3.8]. To show the next result it will be convenient to work over a local ring. Thus, given a point $\lambda_{*}=\left(D_{*}, F_{*}\right)$, we shall denote the local ring of convergent power series at $\lambda_{*}$ by $\mathbb{R}\{D, F\}_{\lambda_{*}}$.

Theorem 3.4. Consider the center at the origin of system (2) with an even $n \geqslant 4$. Then at most one critical period can bifurcate from a nonlinear isochrone, and at most two critical periods can bifurcate from the linear isochrone. Moreover, there are perturbations with exactly one critical period from each nonlinear isochrone, and perturbations with exactly one or exactly two critical periods from the linear isochrone.

Proof. Let us study the case $B \neq 0$ first. Then the homogeneity properties of the parameters $B, D$ and $F$ in the differential system (2) enable us to take $B=1$. We claim that the dehomogenized period coefficients ${ }^{a} p_{2 k}$ for $k \in \mathbb{N}$ are in the ideal ${ }^{a} \mathfrak{m}_{1}=\left({ }^{a} p_{2},{ }^{a} p_{4}\right)$ over the local ring $\mathbb{R}\{D, F\}_{\lambda_{*}}$ localized at any one of the 
isochrones $\lambda_{*} \in\left\{i_{1}, i_{2}, i_{3}\right\}$. To this end we shall use that, by Theorem $3.1,{ }^{a} \mathfrak{m}_{1}=\left({ }^{a} p_{2},{ }^{a} p_{4}\right)$ is radical in $\mathbb{R}[D, F]$, and that

$$
V\left({ }^{a} \mathfrak{m}_{1}\right)=\left\{i_{1}, i_{2}, i_{3}, \ell_{1}, \ell_{2}, \ell_{3}, \ell_{4}, \ell_{5}\right\} .
$$

We take $q \in \mathbb{R}[D, F]$ such that $q\left(i_{j}\right) \neq 0$ for $j=1,2,3$ and $q\left(\ell_{j}\right)=0$ for $j=1,2, \ldots, 5$. (For instance, a possible choice is the polynomial $r_{1}(D)$ in the proof of Theorem 3.1.) Accordingly, since ${ }^{a} p_{2 k}\left(i_{j}\right)=0$ for $j=1,2,3$, we have that $q^{a} p_{2 k} \in{ }^{a} \mathfrak{m}_{1}$ and, consequently, there exist $s_{k, 1}, s_{k, 2} \in \mathbb{R}[D, F]$ such that $q^{a} p_{2 k}=s_{k, 1}{ }^{a} p_{2}+s_{k, 2}{ }^{a} p_{4}$. This equality proves the claim because $q\left(i_{j}\right) \neq 0$ for $j=1,2,3$. Then, on account of the claim and the fact that $\nabla^{a} p_{2}$ and $\nabla^{a} p_{4}$ are linearly independent at $i_{1}, i_{2}$ and $i_{3}$, the assertions concerning the bifurcation from the nonlinear isochrones follow from the Isochrone Bifurcation Theorem in [3, Theorem 2.2]. By applying the same result, from Proposition 3.3 it follows that at most two critical periods can bifurcate from the linear isochrone, i.e., $B=D=F=0$. Note finally that, since $p_{2}$ and $p_{4}$ are homogeneous polynomials, in each neighbourhood of $(B, D, F)=(0,0,0)$ there exist weak centers of order 1 and 2 . This easily implies that there are perturbations of the linear isochrone with exactly one or exactly two critical periods.

\section{Proof of Theorem B}

In this case by Proposition 2.6 we have that $P(h)=2 \pi+\sum_{k \geqslant 1} p_{k} h^{\frac{k(n-1)}{2}}$, and we will show that the vanishing of $p_{1}$ and $p_{2}$ already yields to the isochrones.

Theorem 4.1. Consider the differential system (2) with an odd $n \geqslant 3$. Then the following hold:

(a) The center is isochronous if, and only if, either $B=D=F=0$, or $B \neq 0$ and $\left(\frac{D}{B}, \frac{F}{B}\right) \in\left\{i_{1}, i_{2}\right\}$.

(b) A weak center which is not an isochrone has at most order one. Moreover there are perturbations of the weak centers of order one with one critical period.

(c) The ideal $\mathfrak{m}_{3}=\left(p_{1}, p_{2}\right)$ is radical over the ring $\mathbb{R}[B, D, F]$. In particular, $p_{k} \in \mathfrak{m}_{3}$ for all $k \geqslant 1$.

Proof. In this case the first non-identically zero period coefficient is linear in the parameters. Indeed, see the Appendix, up to a positive scalar factor is given by

$$
p_{1}=B-n D-F \text {, }
$$

so that, contrary to the case $n$ even, there are also weak centers of finite order with $B=0$. We compute a Gröbner basis for the ideal $\mathfrak{m}_{3}=\left(p_{1}, p_{2}\right)$ relative to the lexicographic order given by $B>F>D$, and it follows that $\mathfrak{m}_{3}=\left(q, p_{1}\right)$ with

$$
q=\left((n-1) F+D n^{2}\right) D .
$$

On account of this, the common roots of $p_{1}$ and $p_{2}$ are given by $\{B=0, D=0, F=0\},\{D=0, F=B\}$ and $\left\{D=-\frac{n-1}{n} B, F=n B\right\}$. The first one is the linear isochrone. By [2, Theorem 2.1] the other two are isochrones as well. This proves $(a)$ and that a weak center which is not an isochrone has at most order one. The last assertion in $(b)$ is clear as well because $p_{1}$ changes its sign in each neighbourhood of $p_{1}=0$.

Note at this point that $V\left({ }^{a} \mathfrak{m}_{3}\right)=\left\{i_{1}, i_{2}\right\}$. One can also check that the Jacobian matrix of the mapping $(D, F) \longmapsto\left({ }^{a} p_{1}(D, F),{ }^{a} p_{2}(D, F)\right)$ has rank 2 at $i_{1}$ and $i_{2}$. Accordingly, by the Exponent One Lemma in [3, Lemma A.1], the exponents in the primary decomposition of ${ }^{a} \mathfrak{m}_{3}$ at $i_{1}$ and $i_{2}$ are both 1 . Thus, Noether's Fundamental Theorem (see [18, pages $61-65]$ ) shows that ${ }^{a} \mathfrak{m}_{3}=\mathfrak{q}_{1} \cap \mathfrak{q}_{2}$, where

$$
\mathfrak{q}_{1}:=\left({ }^{a} \mathfrak{m}_{3}, D, F-1\right)=(D, F-1) \text { and } \mathfrak{q}_{2}:=\left({ }^{a} \mathfrak{m}_{3}, n D+n-1, F-n\right)=(n D+n-1, F-n) .
$$

Therefore (see [20, pages 180-182]) we can assert that $\mathfrak{m}_{3} \subset{ }^{h}\left({ }^{a} \mathfrak{m}_{3}\right)={ }^{h} \mathfrak{q}_{1} \cap{ }^{h} \mathfrak{q}_{2}$ and

$$
{ }^{h} \mathfrak{q}_{1}=(D, F-B) \text { and }{ }^{h} \mathfrak{q}_{2}=(n D+(n-1) B, F-n B)
$$


In this case one can verify (for instance, by means of the Gröbner package of Maple) that in fact we have $\mathfrak{m}_{3}={ }^{h}\left({ }^{a} \mathfrak{m}_{3}\right)={ }^{h} \mathfrak{q}_{1} \cap{ }^{h} \mathfrak{q}_{2}$. Hence, since ${ }^{h} \mathfrak{q}_{1}$ and ${ }^{h} \mathfrak{q}_{2}$ are prime, by Theorem 5 in [19, page 209] it follows that $\mathfrak{m}_{3}$ is radical. This proves $(c)$ and concludes the proof of the result.

Following the referee's suggestion, let us mention that the radicality of $\mathfrak{m}_{3}$ can be checked directly with the computer algebra system Singular by means of the routine primdecGTz(), which is based on an algorithm from [9].

Theorem 4.2. Consider the center at the origin of system (2) with an odd $n \geqslant 3$. Then at most one critical period can bifurcate from an isochrone. Moreover, there are perturbations with exactly one critical period from each isochrone.

Proof. The upper bound follows from $(c)$ in Theorem 4.1 by applying the Isochrone Bifurcation Theorem in $\left[3\right.$, Theorem 2.2]. Since one can check that $\nabla p_{1}$ and $\nabla p_{2}$ are linearly independent at each nonlinear isochrone, the same result shows that we can perturb them to obtain one critical period. For the linear isochrone this fact follows by using that in each neighbourhood of $(B, D, F)=(0,0,0)$ there exist a weak center of order one. Hence the result is proved.

It is clear from Theorems A and B that the bifurcation of local critical periods from the center is much simpler for $n$ odd than $n$ even. One may conjecture that the period function of the center at the origin of the generalized Loud's system for $n$ odd is either monotonic or it has one critical period. This makes more affordable the problem of obtaining the bifurcation diagram of the period function for $n$ odd. This diagram consists of three curves, each one related to the bifurcation in $(a),(b)$ and $(c)$ that we enumerate at the beginning of the paper. The first one is the plane $p_{1}=0$. If the conjecture is true, then the second one does not exist. Thus the first step to solve the problem will be to locate the third one by using the tools developed in [13]. We hope to tackle this in a subsequent work.

\section{Appendix}

\section{Expression of the coefficients in Lemma 2.5}

$$
\begin{aligned}
\beta_{1}= & -\alpha_{1}, \\
\beta_{2}= & \alpha_{1}{ }^{2}(k+1)-\alpha_{2}, \\
\beta_{3}= & \left(-3 / 2 \alpha_{1}{ }^{3}(k+1)+3 \alpha_{2} \alpha_{1}\right)(k+2 / 3)-\alpha_{3}, \\
\beta_{4}= & \left(\left(8 / 3 \alpha_{1}{ }^{4}(k+1)-8 \alpha_{2} \alpha_{1}^{2}\right)(k+3 / 4)+2 \alpha_{2}^{2}+4 \alpha_{1} \alpha_{3}\right)(k+1 / 2)-\alpha_{4}, \\
\beta_{5}= & \left(\left(-125 / 24 \alpha_{1}{ }^{5}(k+1)+125 / 6 \alpha_{2} \alpha_{1}{ }^{3}\right)(k+4 / 5)-25 / 2 \alpha_{2}{ }^{2} \alpha_{1}-25 / 2 \alpha_{3} \alpha_{1}{ }^{2}\right)(k+3 / 5) \\
& \left.+5 \alpha_{4} \alpha_{1}+5 \alpha_{3} \alpha_{2}\right)(k+2 / 5)-\alpha_{5}, \\
\beta_{6}= & \left(\left(\left(54 / 5 \alpha_{1}{ }^{6}(k+1)-54 \alpha_{2} \alpha_{1}{ }^{4}\right)(k+5 / 6)+54 \alpha_{2}{ }^{2} \alpha_{1}{ }^{2}+36 \alpha_{3} \alpha_{1}{ }^{3}\right)(k+2 / 3)\right. \\
& \left.\left.-36 \alpha_{1} \alpha_{3} \alpha_{2}-6 \alpha_{2}{ }^{3}-18 \alpha_{4} \alpha_{1}{ }^{2}\right)(k+1 / 2)+3 \alpha_{3}{ }^{2}+6 \alpha_{4} \alpha_{2}+6 \alpha_{5} \alpha_{1}\right)(k+1 / 3)-\alpha_{6} .
\end{aligned}
$$

\section{Expression of the coefficients in Proposition 2.6}

For each $i=1,2, \ldots, 6$, we have that $d_{i}=\widehat{d}_{i} / s_{i}$ where $s_{i}$ is a polynomial on $n$ which is positive for $n \geqslant 1$ and $\widehat{d}_{i}$ is given by

$\widehat{d_{1}}=B-n D-F$, 


$$
\begin{aligned}
& \widehat{d}_{2}=\left(-n^{2}+3 n+1\right) B^{2}+\left(2 n^{3}-5 n^{2}+1\right) B D+\left(n^{2}-3-8 n\right) B F+\left(-n+4 n^{3}\right) D^{2} \\
& +\left(-2+8 n^{2}\right) D F+(5 n+2) F^{2}, \\
& \widehat{d_{3}}=\left(2 n^{4}-12 n^{3}+15 n^{2}+7 n-2\right) B^{3}+\left(-6 n^{5}+31 n^{4}-42 n^{3}-2 n^{2}+15 n-2\right) B^{2} D \\
& +\left(-2 n^{4}+30 n^{3}-67 n^{2}-31 n+8\right) B^{2} F+\left(-27 n^{5}+54 n^{4}-6 n^{3}-21 n^{2}+6 n\right) B D^{2} \\
& +\left(-45 n^{4}+129 n^{3}-3 n^{2}-51 n+6\right) B D F+\left(-18 n^{3}+95 n^{2}+43 n-10\right) B F^{2} \\
& +\left(-27 n^{5}+9 n^{4}+12 n^{3}-4 n^{2}\right) D^{3}+\left(-81 n^{4}+27 n^{3}+36 n^{2}-12 n\right) D^{2} F \\
& +\left(-93 n^{3}+11 n^{2}+40 n-4\right) D F^{2}+\left(-43 n^{2}-19 n+4\right) F^{3}, \\
& \widehat{d}_{4}=\left(-36 n^{7}+326 n^{6}-925 n^{5}+697 n^{4}+439 n^{3}-297 n^{2}-3 n+9\right) B^{4} \\
& +\left(144 n^{8}-1148 n^{7}+3172 n^{6}-3058 n^{5}-436 n^{4}+1686 n^{3}-438 n^{2}-60 n+18\right) B^{3} D \\
& +\left(36 n^{7}-784 n^{6}+3710 n^{5}-4360 n^{4}-2250 n^{3}+1690 n^{2}+36 n-54\right) B^{3} F \\
& +\left(1056 n^{8}-4560 n^{7}+5766 n^{6}-513 n^{5}-2973 n^{4}+1422 n^{3}-24 n^{2}-75 n+9\right) B^{2} D^{2} \\
& +\left(1568 n^{7}-8992 n^{6}+13902 n^{5}-46 n^{4}-7672 n^{3}+2124 n^{2}+318 n-90\right) B^{2} D F \\
& +\left(458 n^{6}-4813 n^{5}+9699 n^{4}+4166 n^{3}-3472 n^{2}-111 n+117\right) B^{2} F^{2} \\
& +\left(2304 n^{8}-5184 n^{7}+1808 n^{6}+2468 n^{5}-1754 n^{4}+154 n^{3}+102 n^{2}-18 n\right) B D^{3} \\
& +\left(6336 n^{7}-16608 n^{6}+5604 n^{5}+8442 n^{4}-5130 n^{3}+174 n^{2}+282 n-36\right) B D^{2} F \\
& +\left(6096 n^{6}-19572 n^{5}+3114 n^{4}+11070 n^{3}-3270 n^{2}-534 n+144\right) B D F^{2} \\
& +\left(2028 n^{5}-9178 n^{4}-3326 n^{3}+3074 n^{2}+126 n-108\right) B F^{3} \\
& +\left(1536 n^{8}-1280 n^{7}-704 n^{6}+800 n^{5}-106 n^{4}-45 n^{3}+9 n^{2}\right) D^{4} \\
& +\left(6144 n^{7}-5120 n^{6}-2816 n^{5}+3200 n^{4}-424 n^{3}-180 n^{2}+36 n\right) D^{3} F \\
& +\left(10176 n^{6}-7008 n^{5}-5388 n^{4}+4338 n^{3}-198 n^{2}-264 n+36\right) D^{2} F^{2} \\
& +\left(8512 n^{5}-2896 n^{4}-5096 n^{3}+1620 n^{2}+276 n-72\right) D F^{3} \\
& +\left(3142 n^{4}+971 n^{3}-995 n^{2}-48 n+36\right) F^{4}, \\
& \widehat{d}_{6}=\left(22356000 n^{12}-52455600 n^{11}+26071200 n^{10}+29946600 n^{9}-37547670 n^{8}+9582565 n^{7}+5182735 n^{6}\right. \\
& \left.-3652725 n^{5}+674995 n^{4}+28660 n^{3}-21960 n^{2}+1800 n\right) F^{2} D^{4} \\
& +\left(32239080 n^{10}-58784940 n^{9}+7218330 n^{8}+45850095 n^{7}-28856265 n^{6}-1853565 n^{5}+6301515 n^{4}\right. \\
& \left.-1632090 n^{3}-11040 n^{2}+44520 n-3600\right) F^{4} D^{2} \\
& +\left(1458000 n^{14}-10432800 n^{13}+29127600 n^{12}-37494180 n^{11}+14316975 n^{10}+18675875 n^{9}-25071500 n^{8}\right. \\
& \left.+9676590 n^{7}+1572250 n^{6}-2546930 n^{5}+785850 n^{4}-59610 n^{3}-15025 n^{2}+3055 n-150\right) B^{3} D^{3} \\
& +\left(34214400 n^{11}-73664640 n^{10}+26805600 n^{9}+48733200 n^{8}-47357160 n^{7}+6839860 n^{6}+8240140 n^{5}\right. \\
& \left.-3995500 n^{4}+461460 n^{3}+77480 n^{2}-20840 n+1200\right) F^{3} D^{3} \\
& +\left(295920 n^{14}-2904120 n^{13}+11550840 n^{12}-23191710 n^{11}+22259355 n^{10}-2407965 n^{9}-15455940 n^{8}\right. \\
& \left.+13772475 n^{7}-3200085 n^{6}-1671285 n^{5}+1186815 n^{4}-232860 n^{3}-2355 n^{2}+5565 n-450\right) B^{4} D^{2} \\
& +\left(71448 n^{12}-1495538 n^{11}+10150867 n^{10}-28945763 n^{9}+30647264 n^{8}+5904293 n^{7}-26745533 n^{6}\right. \\
& \left.+10559847 n^{5}+2698327 n^{4}-2222886 n^{3}+276245 n^{2}+29715 n-4950\right) B^{4} F^{2} \\
& +\left(5313690 n^{9}-24943107 n^{8}+9016935 n^{7}+18805234 n^{6}-11322326 n^{5}-1806069 n^{4}+2134129 n^{3}\right. \\
& \left.-260810 n^{2}-36180 n+5400\right) B F^{5} \\
& +\left(3499200 n^{14}-12830400 n^{13}+15322608 n^{12}-1662768 n^{11}-11463228 n^{10}+9517212 n^{9}-1600539 n^{8}\right. \\
& \left.-1525977 n^{7}+900018 n^{6}-158772 n^{5}-7329 n^{4}+5385 n^{3}-450 n^{2}\right) B D^{5} \\
& +\left(3304800 n^{14}-17178480 n^{13}+32812560 n^{12}-23071320 n^{11}-8687130 n^{10}+24486665 n^{9}-13783400 n^{8}\right.
\end{aligned}
$$


$\left.+329065 n^{7}+2834170 n^{6}-1213010 n^{5}+159415 n^{4}+16730 n^{3}-6315 n^{2}+450 n\right) B^{2} D^{4}$

$+\left(2448330 n^{10}-22177325 n^{9}+51747300 n^{8}-9271595 n^{7}-39932110 n^{6}+20857250 n^{5}+3922855 n^{4}\right.$

$\left.-4061810 n^{3}+498425 n^{2}+64380 n-9900\right) B^{2} F^{4}$

$+\left(3580200 n^{13}-28729620 n^{12}+88178130 n^{11}-121012635 n^{10}+45818385 n^{9}+65694000 n^{8}-81082350 n^{7}\right.$

$\left.+25011690 n^{6}+8450370 n^{5}-7541610 n^{4}+1588380 n^{3}+13185 n^{2}-38955 n+3150\right) B^{3} F D^{2}$

$+\left(10640880 n^{11}-74974020 n^{10}+165761610 n^{9}-88172260 n^{8}-97582080 n^{7}+116367560 n^{6}-19489620 n^{5}\right.$

$\left.-18635460 n^{4}+7965500 n^{3}-517400 n^{2}-154770 n+17100\right) B^{2} F^{3} D$

$+\left(18052272 n^{9}-23713800 n^{8}-7150044 n^{7}+20645566 n^{6}-5469026 n^{5}-3159954 n^{4}+1580110 n^{3}\right.$

$\left.-101420 n^{2}-33720 n+3600\right) F^{5} D$

$+\left(12052800 n^{13}-67398480 n^{12}+135303480 n^{11}-96660540 n^{10}-39329250 n^{9}+103590660 n^{8}-53554400 n^{7}\right.$

$\left.-1930280 n^{6}+11727180 n^{5}-4176980 n^{4}+376420 n^{3}+77520 n^{2}-17430 n+900\right) B^{2} F D^{3}$

$+\left(21373740 n^{10}-91779660 n^{9}+77203605 n^{8}+46485375 n^{7}-78719610 n^{6}+16858920 n^{5}+12322695 n^{4}\right.$

$\left.-5676495 n^{3}+364530 n^{2}+116220 n-12600\right) B F^{4} D$

$+\left(2778300 n^{12}-26645940 n^{11}+93688785 n^{10}-136330915 n^{9}+39093480 n^{8}+91280230 n^{7}-83285470 n^{6}\right.$

$\left.+10281390 n^{5}+13666150 n^{4}-5449650 n^{3}+360645 n^{2}+99525 n-11250\right) B^{3} F^{2} D$

$+\left(379368 n^{13}-4534908 n^{12}+21491052 n^{11}-49585638 n^{10}+51413464 n^{9}-2927352 n^{8}-39446228 n^{7}\right.$

$\left.+28809142 n^{6}-2316878 n^{5}-4864886 n^{4}+1815690 n^{3}-122510 n^{2}-31020 n+3600\right) B^{4} F D$

$+\left(34408800 n^{12}-135600480 n^{11}+160632720 n^{10}-3292560 n^{9}-130387770 n^{8}+90362410 n^{7}-5321240 n^{6}\right.$

$\left.-17418900 n^{5}+7198570 n^{4}-743930 n^{3}-133680 n^{2}+33060 n-1800\right) B F^{2} D^{3}$

$+\left(37098000 n^{11}-151412760 n^{10}+163342260 n^{9}+28381350 n^{8}-148360830 n^{7}+73525080 n^{6}+9996480 n^{5}\right.$

$\left.-17424270 n^{4}+4202190 n^{3}+32400 n^{2}-111300 n+9000\right) B F^{3} D^{2}$

$+\left(16891200 n^{12}-103539600 n^{11}+217819680 n^{10}-146695620 n^{9}-85647420 n^{8}+169241730 n^{7}\right.$

$\left.-66530700 n^{6}-14785080 n^{5}+17473050 n^{4}-3938280 n^{3}-30390 n^{2}+100170 n-8100\right) B^{2} F^{2} D^{2}$

$+\left(16912800 n^{13}-64482480 n^{12}+78317280 n^{11}-7822440 n^{10}-59368590 n^{9}+47580765 n^{8}-6830265 n^{7}\right.$

$\left.-7926570 n^{6}+4251990 n^{5}-666285 n^{4}-46815 n^{3}+23610 n^{2}-1800 n\right) B F D^{4}$

$+\left(1399680 n^{14}-3405888 n^{13}+1889568 n^{12}+1792368 n^{11}-2533032 n^{10}+764964 n^{9}+305142 n^{8}\right.$

$\left.-256883 n^{7}+53737 n^{6}+1109 n^{5}-1675 n^{4}+150 n^{3}\right) D^{6}$

$+\left(4779942 n^{8}-2706785 n^{7}-3599599 n^{6}+2510141 n^{5}+334669 n^{4}-459344 n^{3}\right.$

$\left.+56040 n^{2}+8240 n-1200\right) F^{6}$

$+\left(-3600 n^{13}+56208 n^{12}-349998 n^{11}+1091367 n^{10}-1700148 n^{9}+921286 n^{8}+706463 n^{7}-1075462 n^{6}\right.$

$\left.+316778 n^{5}+110821 n^{4}-74480 n^{3}+9350 n^{2}+805 n-150\right) B^{6}$

$+\left(3600 n^{13}-127656 n^{12}+1262786 n^{11}-5488399 n^{10}+11257251 n^{9}-8601065 n^{8}-3693801 n^{7}\right.$

$\left.+8424060 n^{6}-2883060 n^{5}-857813 n^{4}+637311 n^{3}-79685 n^{2}-7695 n+1350\right) B^{5} F$

$+\left(21600 n^{14}-305928 n^{13}+1800168 n^{12}-5584832 n^{11}+9386893 n^{10}-7074051 n^{9}-1408233 n^{8}\right.$

$\left.+6403707 n^{7}-3840228 n^{6}+137774 n^{5}+674375 n^{4}-235755 n^{3}+16155 n^{2}+3765 n-450\right) B^{5} D$

$+\left(582750 n^{11}-8202165 n^{10}+36252295 n^{9}-54551620 n^{8}+44490 n^{7}+44123410 n^{6}-20038630 n^{5}\right.$

$\left.-4402790 n^{4}+4047080 n^{3}-499565 n^{2}-59265 n+9450\right) B^{3} F^{3}$

$+\left(8398080 n^{13}-20435328 n^{12}+11337408 n^{11}+10754208 n^{10}-15198192 n^{9}+4589784 n^{8}+1830852 n^{7}\right.$

$\left.-1541298 n^{6}+322422 n^{5}+6654 n^{4}-10050 n^{3}+900 n^{2}\right) F D^{5}$. 


\section{References}

[1] N. N. Bautin, On the number of limit cycles which appear with the variation of coefficients from an equilibrium position of focus or center type, Amer. Math. Soc. Transl. 100 (1954) 1-19.

[2] I. Boussaada, R. Chouikha, J. Strelcyn, Isochronicity conditions for some planar polynomial systems, Bull. Sci. Math. 135 (2011) 89-112.

[3] C. Chicone and M. Jacobs, Bifurcation of critical periods for plane vector fields, Trans. Amer. Math. Soc. 312 (1989) 433-486.

[4] C. Christopher, An algebraic approach to the classification of centers in polynomial Liénard systems, J. Math. Anal. Appl. 229 (1999) 319-329.

[5] A. Cima, A. Gasull, V. Mañosa and F. Mañosas, Algebraic properties of the Lyapunov and period constants, Rocky Mountain J. Math. 27 (1997) 471-501.

[6] Zhengdong Du, On the critical periods of Liénard systems with cubic restoring forces, Int. J. Math. Math. Sci. 61 (2004) 3259-3274.

[7] A. Gasull, A. Guillamon and J. Villadelprat, The period function for second-order quadratic ODEs is monotone, Qual. Theory Dyn. Syst. 4 (2004) 329-352.

[8] A. Gasull and Jiang Yu, On the critical periods of perturbed isochronous centers, J. Differential Equations 244 (2008) 696-715.

[9] P. Gianni, B. Trager, and G. Zacharias, Gröbner bases and primary decomposition of polynomial ideals, J. Symbolic Comput. 6 (1988) 149-167.

[10] M. Grau and J. Villadelprat, Bifurcation of critical periods from Pleshkan's isochrones, J. Lond. Math. Soc. 81 (2010) 142-160.

[11] M. Han and V. Romanovski, Estimating the number of limit cycles in polynomials systems, J. Math. Anal. Appl. 368 (2010) 491-497.

[12] W. S. Loud, Behavior of the period of solutions of certain plane autonomous systems near centers, Contributions to Differential Equations 3 (1964) 21-36.

[13] M. Mardešić, D. Marín and J. Villadelprat, The period function of reversible quadratic centers, J. Differential Equations 224 (2006) 120-171.

[14] V. G. Romanovski and M. Han, Critical period bifurcations of a cubic system, J. Phys. A: Math. Gen. 36 (2003) 5011-5022.

[15] C. Rousseau and B. Toni, Local bifurcation of critical periods in vector fields with homogeneous nonlinearities of the third degree, Canad. Math. Bull. 36 (1993) 473-484.

[16] C. Rousseau and B. Toni, Local bifurcations of critical periods in the reduced Kukles system, Canad. J. Math. 49 (1997) 338-358.

[17] J. Villadelprat, The period function of the generalized Lotka-Volterra centers, J. Math. Anal. Appl. 341 (2008) 834-854.

[18] B. L. van der Waerden, "Modern algebra, volume II", Ungar, New York, 1950.

[19] O. Zariski and P. Samuel, "Commutative algebra, volume I", Graduate Texts in Mathematics, No. 28, Springer, New York, 1975.

[20] O. Zariski and P. Samuel, "Commutative algebra, volume II", Graduate Texts in Mathematics, No. 29, Springer, New York, 1975. 